\title{
Intense terahertz pulses from SPARC_LAB coherent radiation source
}

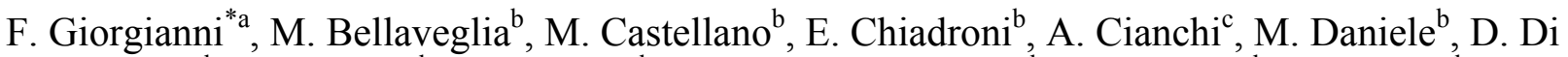 \\ Giovenale $^{\mathrm{b}}$, G. Di Pirro ${ }^{\mathrm{b}}$, M. Ferrario ${ }^{\mathrm{b}}$, S. Lupi ${ }^{\mathrm{a}}$, A. Mostacci ${ }^{\mathrm{d}}$, M. Petrarca ${ }^{\mathrm{d}}$, R. Pompili ${ }^{\mathrm{b}}$, V. \\ Shpakov ${ }^{\mathrm{b}}$, F. Villa ${ }^{\mathrm{b}}$ \\ ${ }^{a}$ INFN and Department of Physics, "Sapienza" University of Rome, Piazzale A. Moro 2, I-00185 \\ Roma, Italy; 'Laboratori Nazionali di Frascati - INFN via Enrico Fermi, 4000044 Frascati, Italy; \\ 'INFN and Department of Physics, "Tor Vergata" University, via della Ricerca Scientifica 1, 00133 \\ Rome, Italy; ${ }^{\mathrm{d}}$ INFN and SBAI Department, "Sapienza" University of Rome, Via A. Scarpa 14, \\ 00161 Rome, Italy Rome, Italy
}

\begin{abstract}
The linac-based Terahertz source at the SPARC_LAB test facility is able to generate highly intense Terahertz broadband pulses via coherent transition radiation (CTR) from high brightness electron beams. The $\mathrm{THz}$ pulse duration is typically down to 100 fs RMS and can be tuned through the electron bunch duration and shaping. The measured stored energy in a single $\mathrm{THz}$ pulse has reached $40 \mu \mathrm{J}$, which corresponds to a peak electric field of $1.6 \mathrm{MV} / \mathrm{cm}$ at the $\mathrm{THz}$ focus. Here we present the main features, in particular spatial and spectral distributions and energy characterizations of the SPARC_LAB THz source, which is very competitive for investigations in Condensed Matter, as well as a valid tool for electron beam longitudinal diagnostics.
\end{abstract}

Keywords: Terahertz Radiation Source, High brightness photo-injector, Electron beam diagnostic.

\section{INTRODUCTION}

Terahertz (THz) radiation (15 THz- $30 \mathrm{GHz}, 20-1000$ microns; 1-60 meV) is a portion of the electromagnetic (e.m.) spectrum, which lies in the gap between Microwave and Infrared. THz-based technologies and research applications have seen a rapid increment in the last years due to the development of new radiation sources based both on lasers and sub-picosecond electron bunches. Nowadays $\mathrm{THz}$ light is ordinarily generated from tabletop laser-based sources by optical rectification or optoelectronic mechanism, like photoconductive process ${ }^{1}$.

Intense single cycle pulses are increasingly used to explore the nonlinear light-matter interaction at $\mathrm{THz}$ frequency. Recently, high intensity $\mathrm{THz}$ sources can reach pulse energies on the level of tens of $\mathrm{uJ}$ by optical rectification of tens of $\mathrm{mJ}$ laser pulses ${ }^{2,3}$. However, photon down-conversion process suffers from insurmountable limitations to generate high power THz pulses, like the damage threshold of the nonlinear crystals and saturation conversion efficiency. Even higher pulse energies and fields can potentially be reached by accelerator-based sources, which are unaffected from optical conversion limitations.

At the SPARC_LAB test facility ${ }^{4}$, highly intense THz radiation is routinely produced as Coherent Transition Radiation (CTR) and Coherent Diffraction Radiation (CDR) emitted by ultra-short ( 100 fs) high-brightness electron bunches. By proper tailoring the longitudinal electron beam distribution, both broadband ${ }^{5}$ and narrowband tunable $\mathrm{THz}$ radiation can be generated ${ }^{6}$.

In the present work, we present the characterization of the spatial and spectral distribution of CTR radiation in the THz region. Transverse spatial profiles have been compared with numerical simulations carried out with THzTransport code ${ }^{7}$. The measured $\mathrm{THz}$ figure of merits, in particular an energy per pulse up to $40 \mu \mathrm{J}$ and pulse duration down to $100 \mathrm{fs}$ RMS, render the SPARC_LAB source one of the most performant source worldwide for nonlinear THz spectroscopy in Condensed Matter Physics. Furthermore, coherent THz radiation is a valid and useful tool for electron beam longitudinal diagnostics, enabling the reconstruction of the bunch longitudinal charge distribution.

Relativistic Plasma Waves and Particle Beams as Coherent and Incoherent Radiation Sources edited by Dino A. Jaroszynski, Proc. of SPIE Vol. 9509, 950900 - C 2015 SPIE CCC code: $0277-786 \mathrm{X} / 15 / \$ 18 \cdot$ doi: $10.1117 / 12.2178970$ 


\section{THEORY}

Any electron in a relativistic bunch emits transition radiation (TR) when crossing the boundary between two media of different dielectric properties ${ }^{8}$. For $\mathrm{N}$ electrons in a bunch the emitted spectral and angular intensity distribution can be written as:

$$
\frac{d^{2} I}{d \omega d \Omega}=\frac{d^{2} I_{s p}}{d \omega d \Omega}[N+N(N-1) \cdot F(\omega)]
$$

where $\frac{d^{2} I_{s p}}{d \omega d \Omega}$ is the intensity produced by a single particle which depends on the specific mechanism of emission, transition radiation (TR) in our case, while $\mathrm{F}(\omega)$ is the longitudinal form factor of the bunch, i.e. the square modulus of Fourier transform (FT) of the longitudinal bunch distribution S(z). At wavelengths smaller than the longitudinal electron bunch size, typically tens or few hundreds of microns, the spectrum is dominated by incoherent emission (proportional to the number of particles $\mathrm{N}$ in the bunch), and $\mathrm{F}(\omega)$ tends to 0 . At longer wavelengths, with respect to the bunch length, emission starts to be coherent (i.e. proportional to $\mathrm{N}^{2}$ ) because each electron emits in phase and the amplitudes add with constructive interference: $F(\omega)$ tends to 1. For bunch length of the order of $100 \mathrm{fs}$ and less, radiation is emitted in the $\mathrm{THz}$ region of the e.m. spectrum.

TR single particle intensity distribution in Eq. 1 generate from a finite size rectangular target can be written as:

$$
\frac{d^{2} I_{s p}}{d \omega d \Omega}=\frac{1}{4 \pi \varepsilon_{0}} \frac{e^{2} \omega^{2}}{4 \pi^{2} c}\left[\left|E_{x}(h, l, \omega, \theta, \gamma)\right|^{2}+\mid E_{y}(h, l, \omega, \theta, \gamma)^{2}\right]
$$

where $E_{x(y)}(h, l, \omega, \theta, \gamma)$ is the electric field component along the x (y) direction (the plane of the target), $\theta$ represents the observation angle, $h$ and $l$ are the vertical and horizontal target dimensions, while $\gamma$ is the electron Lorentz factor. The full expression of the electric field components in Eq. (2) can be found in ref. 9. The effects of finite size target become non-negligible and introduce a suppression of the radiated intensity at low-frequency when the extent of the particle field, which is of the order of $\lambda \gamma$, exceeds the dimension of the radiation target. This is always the case for coherent radiation at $\mathrm{THz}$ frequency.

\section{EXPERIMENTAL}

The SPARC_LAB photoinjector, which consists in a RF Gun, with embedded a copper cathode, and 3 S-Band travelling wave accelerating sections, is able to provide high brightness electron beams boosted up to $170 \mathrm{MeV}$ at $10 \mathrm{~Hz}$ of repetition rate. A schematic layout is shown in Fig. 1a.

Ultra-short electron bunches, as needed for producing coherent radiation in the $\mathrm{THz}$ range, are generated at SPARC_LAB through RF compression in the so-called velocity bunching (VB) regime ${ }^{10,11}$. The electron beam is injected in the first accelerating section at the zero crossing RF field phase. Since the beam is slightly slower than the phase velocity of the RF wave, a longitudinal phase space rotation occurs, based on a time-velocity chirp in the electron bunch, for which electrons in the tail are faster than those in the bunch head. As consequence, the electron beam slips back to phases where the field is accelerating, but it is simultaneously chirped and compressed.

The so generated ultra-short, $\sim 100 \mathrm{fs}$, and highly charged, up to $\sim 600 \mathrm{pC}$, electron bunches reach the $\mathrm{THz}$ station placed at the end of the linac, as reported in Fig 1a. A diagnostic transfer line, right after the THz station, and equipped with a RF deflecting cavity (RFD), quadrupoles and a dipole magnet, allows a full 6D characterization of the electron beam.

Figure $1 \mathrm{~b}$ reports an experimental scheme of the THz source chamber. In our case, TR is produced on a $30 \times 30 \mathrm{~mm}$ aluminum coated silicon plate placed at $45^{\circ}$ with respect to the electron beam direction, which both generates radiation and reflects it out from the beamline.

The backward-generated radiation, passing through a quartz window, is collected and collimated by a $90^{\circ}$ off axis parabolic mirror with a focal length of $152 \mathrm{~mm}$ and carried to the experimental table by a flat mirror. The coherent radiation spectrum is measured using a customized Michelson interferometer with a $24 \mu \mathrm{m}$ Mylar pellicle beamsplitter coupled with a room-temperature pyroelectric detector (Gentec-EO). The THz total energy per pulse was measured by a further pyroeletric detector, collecting radiation selected and focalized by an off axis parabolic mirror (APM1) mounted on a motorized stage. The transverse spatial distribution of CTR has been observed by means of a THz camera (Spiricon 
Camera III, Ophir) placed in the focal plane of an off axis parabolic mirror (APM2) with a focal length of $100 \mathrm{~mm}$. THz filters $(0.5,1$ and $3 \mathrm{THz})$, with approximately a $15 \%$ bandwidth, have been used to select different frequencies.

The measurements presented in this paper have been performed with a $530 \mathrm{pC}, 110 \mathrm{MeV}$ electron beam with different bunch length to explore different emission spectra.

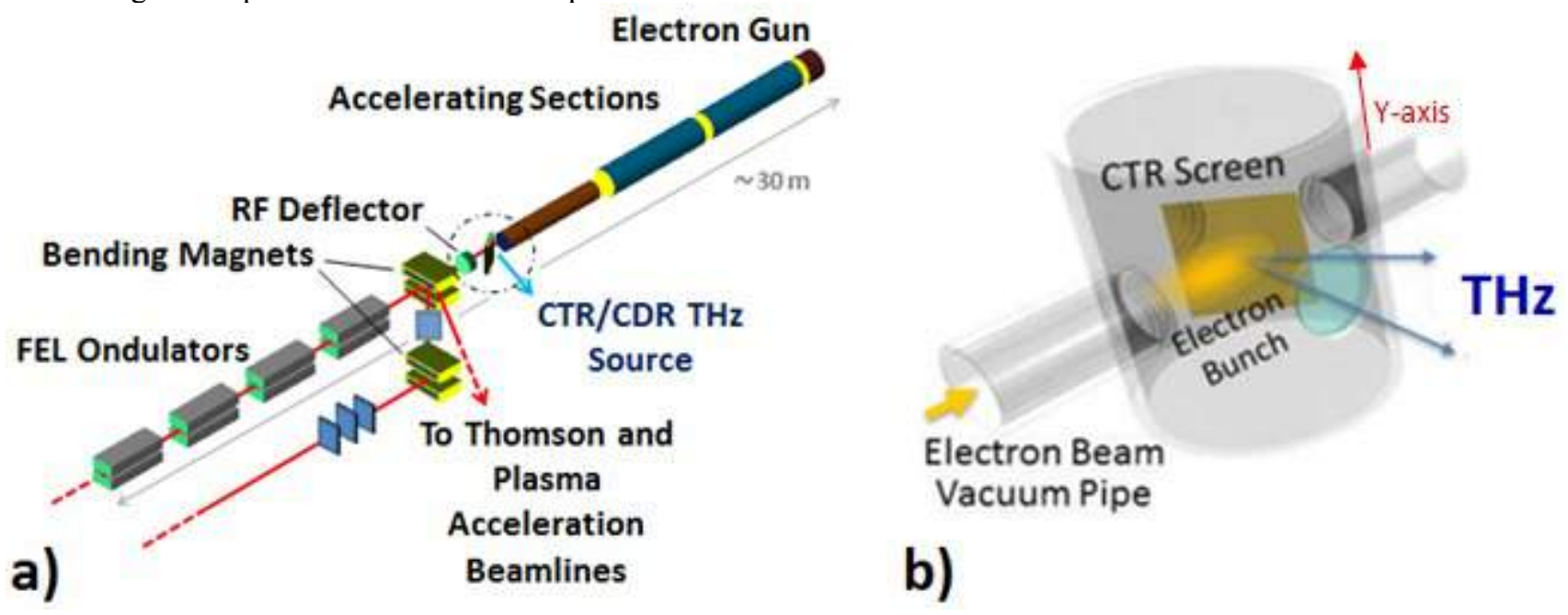

Figure 1: a) Simplified cartoon of SPARC_LAB. b) Experimental layout of the THz source chamber placed at the end of the linac.

\section{RESULTS}

Figure 2 shows two interferograms of CTR emitted by an electron bunch under different compression regimes, characterized by the so-called compression factor, $\mathrm{C}$, defined as the ratio between the uncompressed, measured, bunch length and the bunch length measured after compression. The uncompressed beam has a duration of $6 \mathrm{ps} \mathrm{FWHM.} \mathrm{At} \mathrm{the}$ phase of maximum compression, occurring at $-90 \mathrm{deg}$ from the on crest phase (i.e. the phase of maximum energy gain), we measured with the RFD cavity a pulse duration of $253 \mathrm{fs} F W H M$, corresponding to a compression factor $\mathrm{C}_{\mathrm{High}}=24$. Moving back of 4 deg towards the on crest phase, the electron beam shows a lower compression factor, $C_{\text {low }}=9$, being its FWHM time duration of 644 fs.. A lower compression factor, i.e. a longer electron bunch, results in a considerable broadening of the Michelson interferogram. The spectra reported in Figure $2 \mathrm{~b}$ show the change in spectral content, extending up to $3 \mathrm{THz}$ for the higher (red curve) bunch compression. Periodic modulations, present in both spectra, we attribute to Fabry-Pérot multiple reflections interference in the layers of the pyroelectric detector and the dip around 0.5 $\mathrm{THz}$ due to a decrease of pyroelectric detector responsivity. The spectrum can be used to reconstruct the temporal profile of the bunch, evaluating the form factor from Eq. 1, and numerically solving Eq. 2, which takes into account the finite size of the screen. In our case, this effect becomes significant and suppresses low-frequency components below $0.5 \mathrm{THz}$. To compensate the spectra for the losses discussed above, we introduce a low frequency Gaussian reconstruction as reported in ref. 12. The electron bunch profile, $\mathrm{S}(\mathrm{z})$, is retrieved from the form factor, applying Kramers-Kroenig relations ${ }^{13}$. Reconstructed electron bunch profiles for $\mathrm{C}_{\mathrm{High}}$ (red curve) and $\mathrm{C}_{\text {low }}$ (blue curve) are shown in the inset of Fig. 2b.The retrieved FWHM pulse duration is $235 \mathrm{fs}$ and $610 \mathrm{fs}$ for maximum and low compressions respectively, in good agreement with direct measurements by means of RFD cavity.

In the case of maximum compression we have characterized the spatial distribution of focalized CTR at different wavelengths. In Fig. 3a we show a scan of the CTR peculiar annular distribution at $1 \mathrm{THz}$ as a function of distance from APM2 focal plane. Each image has a dimension of $12 \times 12 \mathrm{~mm}$.

The y-component of the spatial distributions in the focal plane at $0.5,1$ and $3 \mathrm{THz}$ is shown in Fig. 3b), c), d) respectively. We observe an overall reduction of the intensity at $3 \mathrm{THz}$ with respect to $1 \mathrm{THz}$ due to the high frequency cut-off, in the form factor $F(\omega)$, given by longitudinal bunch size. At $0.5 \mathrm{THz}$ instead the intensity reduction might be due to both a reduction of sensor sensitivity of the THz camera and the low frequency cut-off introduced by the finite target size. We attribute the spatial transverse asymmetry at $3 \mathrm{THz}$ to optical misalignment, which affects the high frequency.

The experimental data have been compared with numerical calculation using THzTransport code, blue dotted lines in Fig. 3b), c), d). Solving numerically Eq. 2 at fixed frequency the code evaluates the propagation of the THz beam using 
Fourier transformation optics through the optical element, including the quartz window. Peaks position and width are in agreement with experimental data, while their intensity is normalized to experimental values.

We measured a total THz CTR energy per pulse of $40 \mathrm{uJ}$ using a pyroelectric detector for a $530 \mathrm{pC}$ electron beam with a time duration of $200 \mathrm{fs}$ FWHM. This value corresponds to an electric peak field amplitude of $1.6 \mathrm{MV} / \mathrm{cm}$ evaluated on a focal spot size with a diameter of $2 \mathrm{~mm}$.

\section{CONCLUSION}

Intense THz radiation is currently generated the SPARC_LAB facility as coherent transition radiation from ultra-short electron bunches. We have measured both the spatial and spectral distribution of CTR in the THz range and its energy per pulse. Beyond beam longitudinal diagnostic applications, the features shown here render this source very interesting to study peculiar nonlinear processes in condensed matter physics at $\mathrm{THz}$ frequency.

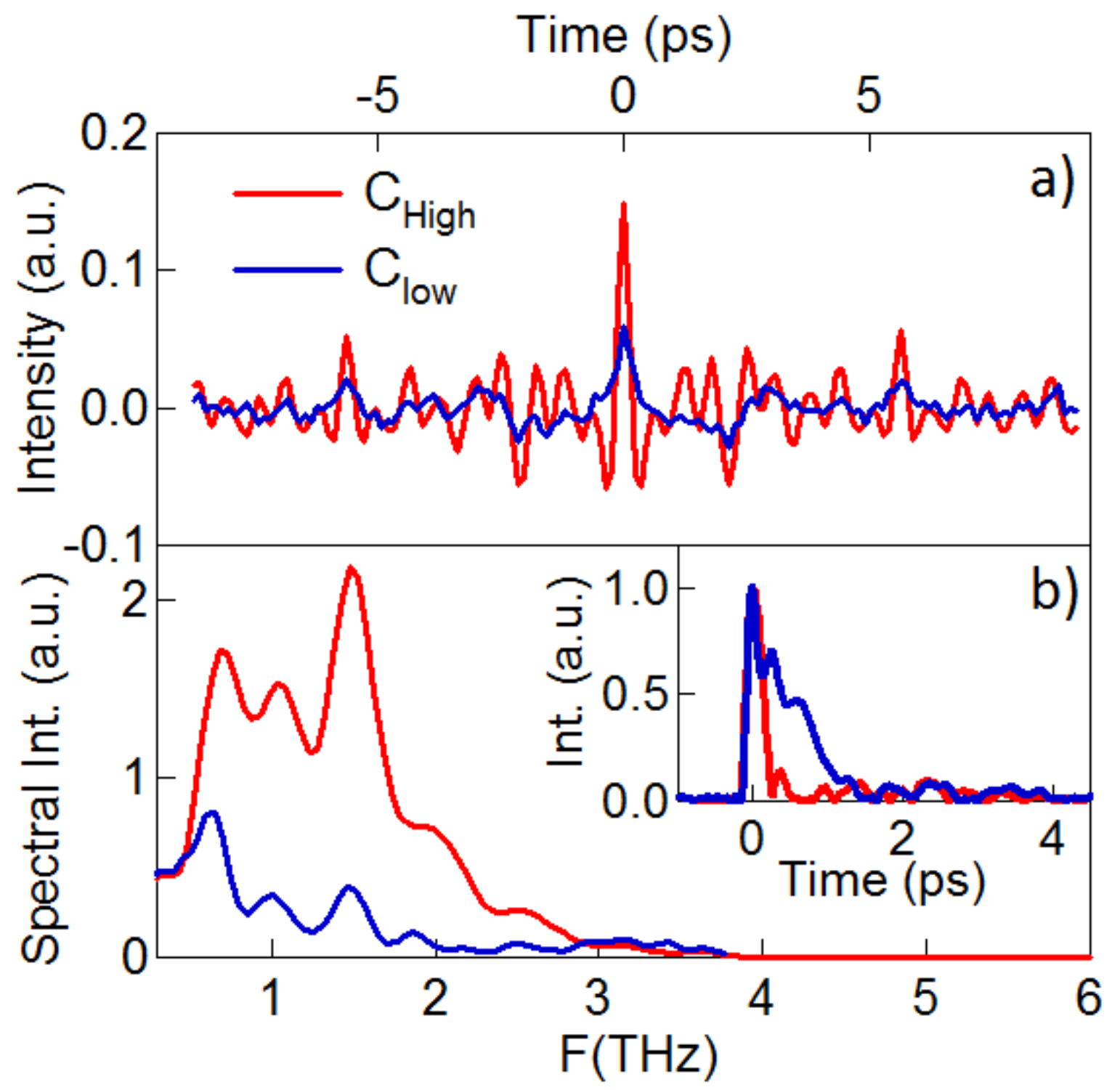

Figure 2 a) Michelson interferograms of CTR radiation from a $530 \mathrm{pC}$ electron beam: $\mathrm{C}_{\mathrm{High}}=24$ in maximum compression (red curve) and $\mathrm{C}_{\text {low }}=9$ (blue curve). b) Spectral distribution. Inset: Reconstruction of electron bunch longitudinal profiles at maximum (red curve) and low (blue) compression factor. 

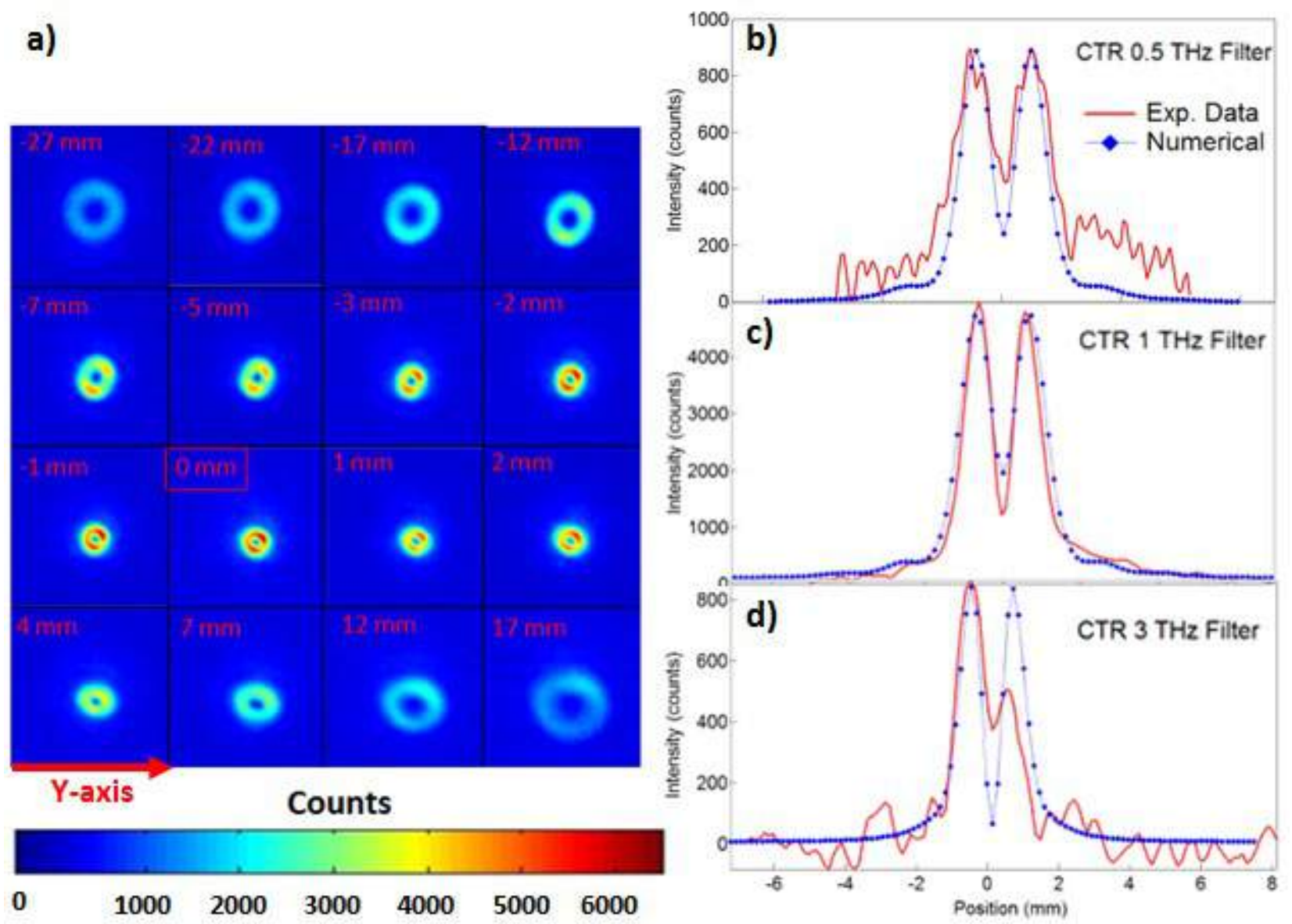

Figure 3 Coherent transition radiation transverse distribution in the focal plane: a) Spatial distribution of the source with a $1 \mathrm{THz}$ filter at different distances from the focal plane $(0 \mathrm{~mm})$. Each frame has a dimension of $12 \times 12 \mathrm{~mm} ; \mathrm{b}), \mathrm{c})$, d) Experimental data (red lines) and numerical calculation (blue dotted lines) of transverse profile at respectively $0.5,1$ and $3 \mathrm{THz}$ on focus. Numerical curves have been normalized.

\section{REFERENCES}

[1] Lee, Y. R., [Principles of Terahertz Science and Technology], Springer Science+Business Media LLC, New York, 59-74 (2009)

[2] Yeh, K. L., Hoffmann, M. C., Hebling, J., \& Nelson, K. A., "Generation of $10 \mu \mathrm{J}$ ultrashort terahertz pulses by optical rectification," Applied Physics Letters, 90(17), 171121-171121 (2007).

[3] Vicario, C., Monoszlai, B., \& Hauri, C. P. "GV/m single-cycle terahertz fields from a laser-driven large-size partitioned organic crystal," Physical Review Letters, 112(21), 213901 (2014).

[4] Ferrario, M. et al. "SPARC_LAB present and future," Nuclear Instruments and Methods in Physics Research B, 309, 183-188 (2013).

[5] Chiadroni, E., et al., "The SPARC linear accelerator based terahertz source," Appl. Phys. Lett., 102(9), 094101 (2013).

[6] Chiadroni, E. et al. "Characterization of the THz radiation source at the Frascati linear accelerator", Rev. Sci. Instrum. 84, 022703 (2013).

[7] Schmidt, B., "THz Transport: Uebergangs und Diffraktionsstrahlung Erzeugung und Transport durch optische Elemente," www.desy.de/ schmidtb

[8] Landau, L. D., Lifschitz, E. M., [Electrodynamics of Continuous Media] Pergamon Press, Oxford (1984). 
[9] Castellano, M., Cianchi, A., Orlandi, G. and Verzilov, V., "Effect of diffraction and target finite size on coherent transition radiation spectra in bunch length measurement," Nuclear Instruments and Method in Physics Research A, 435, 297-307, (1999).

[10] Serafini, L.,"Velocity bunching in photo-injectors," Proc. AIP, 87-106 (2001).

[11] Ferrario, M. et al., "Experimental Demonstration of Emittance Compensation with Velocity Bunching," Phys. Rev. Lett. 104, 054801 (2010).

[12] Wu, Z., et al. "Intense terahertz pulses from SLAC electron beams using coherent transition radiation," Rev. Scientific Instruments,84(2), 022701 (2013).

[13] Lai, R., Happek, U., and Sievers, A. J., "Measurement of the longitudinal asymmetry of a charged particle bunch from the coherent synchrotron or transition radiation spectrum," Phys. Rev. E, 50(6), R4294 (1994). 